\title{
Feasibility of depth sensors to study breast deformation during mammography procedures
}

\author{
Oliver Díaz $^{1 *}$, Arnau Oliver ${ }^{1}$, Sergi Ganau ${ }^{2}$, Eloy García ${ }^{1}$, Joan Martí1 ${ }^{1}$, Melcior Sen- \\ tís $^{2}$, and Robert Martí ${ }^{1}$ \\ ${ }^{1}$ ViCOROB research institute, University of Girona, Girona, Spain \\ \{oliver.diaz, arnau.oliver, eloy.garcia,joan.marti, robert.marti\} \\ @udg.edu \\ ${ }^{2}$ UDIAT - Centre Diagnòstic - Institute Universitari Parc Taulí - UAB, Sabadell, Spain \\ \{sganau, msentis\}@tauli.cat
}

\begin{abstract}
Virtual clinical trials (VCT) currently represent key tools for breast imaging optimisation, especially in two-dimensional planar mammography and digital breast tomosynthesis. Voxelised breast models are a crucial part of VCT as they allow the generation of synthetic image projections of breast tissue distribution. Therefore, realistic breast models containing an accurate representation of women breasts are needed. Current voxelised breast models show, in their compressed version, a very round contour which might not be representative of the entire population. This work pretends to develop an imaging framework, based on depth cameras, to investigate breast deformation during mammographic compression. Preliminary results show the feasibility of depth sensors for such task, however post-processing steps are needed to smooth the models. The proposed framework can be used in the future to produce more accurate compressed breast models, which will eventually generate more realistic images in VCT.
\end{abstract}

Keywords: Breast imaging, breast compression, mammography, DBT, Microsoft Kinect, virtual clinical trials, breast phantom.

\section{Introduction}

Traditionally, large clinical trials are used to evaluate the performance of new x-ray mammography systems or even to optimise image acquisition parameters (e.g. beam quality, projection angle, detector characterisation, etc.). However, clinical trials involve irradiation of asymptomatic women and, at the same time, the entire process becomes expensive and very time consuming. Furthermore, they require a large amount of specialised personnel, e.g. radiographers, radiologists, etc.

Recently, digital breast tomosynthesis (DBT) has been suggested as a replacement of planar X-ray mammography [1]. Furthermore, current DBT clinical trials (STORM [2], 
OSLO [3] and MBTST [4]) are investigating several approaches to replace conventional mammography in screening or to use DBT as an additional imaging modality. However, certain optimisations in DBT image acquisition parameters require faster, cheaper and radiation-free approaches, e.g. optimal number of projections, projection angle, energy spectra, etc. For these reasons, virtual clinical trials (VCTs) represent a pivotal tool which allow large evaluation using synthetic images, i.e. without radiation.

VCTs highly rely on producing realistic images of the breast. Therefore, a large number of anthropomorphic breast models is needed to provide a population-based approximation of breast images. Frequently, these breast models are originally generated in an uncompressed form. Then, these are deformed to produce a compressed breast shape based on several parameters, including breast density or the mechanical properties of the different tissues among others [5].

Several compressed anthropomorphic breast models are available $[6,7,8]$. Two of these models are shown in figure 1 in their compressed form (sagittal plane), where a very round contour is observed. However, we suspect this contour shape might not be representative to the entire women population. Furthermore, compressed breast shape might be related to tissue properties, i.e. breast glandularity, or even breast size. For these reasons, we investigated tools which would allow future analysis of breast deformation during mammography compression in clinical scenarios.

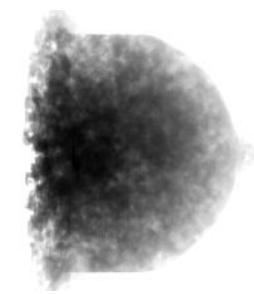

(a)

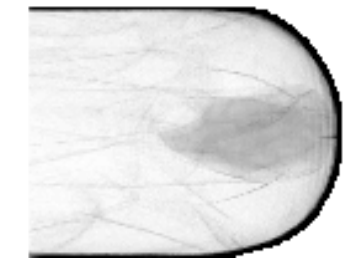

(b)

Fig. 1. Sample of compressed anthropomorphic breast models (sagittal plane) developed by (a) Bliznakova et al [6] and (b) Bakic et al [7]. Note the rounded contour of the breast models in both cases.

Agasthya and Sechopoulos [9] performed a similar study to characterise breast surface during compression using a pair of projector-camera setup. However, we investigate more affordable image sensors (150-200 euros) which provide depth information, as these might represent a low cost approach for this task.

The Microsoft Kinect sensor has been previously used for assessment of the breast for surgery planning and breast volume estimation [10,11,12]. Therefore, it is a good starting point for this investigation. This work presents an imaging framework to study breast deformation during mammography compression. Since the compression procedure is exactly the same, this could be applied to both conventional mammography and DBT. Results of this research can be useful to produce more accurate compressed breast 
models in the future, and therefore, generate more realistic images in VCTs. In addition, accurate knowledge of breast models can also improve scattered radiation estimation since breast curvature plays an important role [13] or even to develop more precise knowledge for mean glandular dose calculations.

The rest of the paper is structured as follows. Section 2 describes the measurements performed to estimate the accuracy of Kinect sensors as well as the clinical setup used to acquired breast contours during mammography image acquisition. The results section shows the precision's analysis of the Kinect sensor and several sample images of uncompressed and compressed breasts from participants. Observations and issues encounter during the Kinect image acquisition are described in the discussion section. Finally, the main outcomes of this work are reported in the conclusion section.

\section{Methodology}

In a first stage, a total of 4 women, of age $45 \pm 4.5$ years old, have volunteered to capture their breast shape during their routinely DBT acquisition at the clinic site. The Kinect for Xbox 360 sensor, Kinect v1, has been employed to image each breast before and after cranio-caudal (CC) compression. The Kinect device not only has an optical sensor to acquire colour images (RGB), but also an infrared emitter and sensor which allow generating images with depth information of the scene, so called RGB-D (RGB-Depth).

The first task undertaken was to quantify the correlation between the Kinect sensor and experimental measurements, and evaluate the Kinect performance for the task of this paper. In order to compare experimentally depth measures with Kinect measures, the following experiment was designed in the laboratory: a piece of cardboard (150x85 $\mathrm{mm}^{2}$ ) was placed at a fixed distance from the Kinect sensor. The distance from the Kinect sensor to the cardboard was varied from 800 to $1200 \mathrm{~mm}$, in steps of $10 \mathrm{~mm}$. The distance produced by the Kinect sensor was calculated as the average distance value of a 10x10 pixel region of interest (ROI) within the cardboard depth image.

Secondly, the Kinect sensor was mounted in a tripod at the clinical site (UDIAT - Centre Diagnòstic - Institute Universitari Parc Taulí - UAB, Sabadell) as shown in figure 2a. The Kinect sensor was located at between 800 and $900 \mathrm{~mm}$ from the DBT/mammography unit, i.e. Hologic Selenia Dimensions. Before entering the image acquisition room, the purpose of this experiment was explained to each of the participants. A sample RGB image of a volunteer obtained from the Kinect point of view is shown in figure $2 \mathrm{~b}$. For the purpose of this work, breasts were imaged before and after compression prior image acquisition. As used in clinical practise at UDIAT, a flexible breast compression paddle was employed. Due to the fixed position of the Kinect sensor, only CC compression was investigated in this work. Three-dimensional (3D) triangular meshes, 3D point clouds and RGBD images of each breast were generated with the aid of the software tools Skanect (v1.8.3), KScan3D (v1.2.0.2) and Matlab (R2015b). 


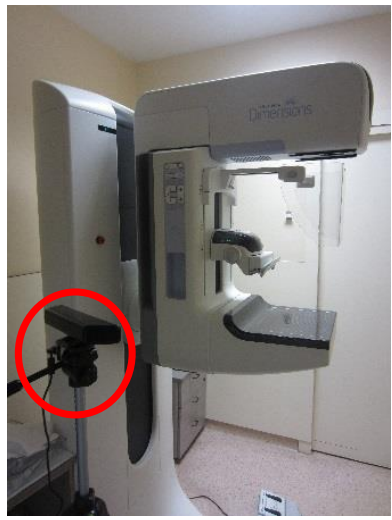

(a)

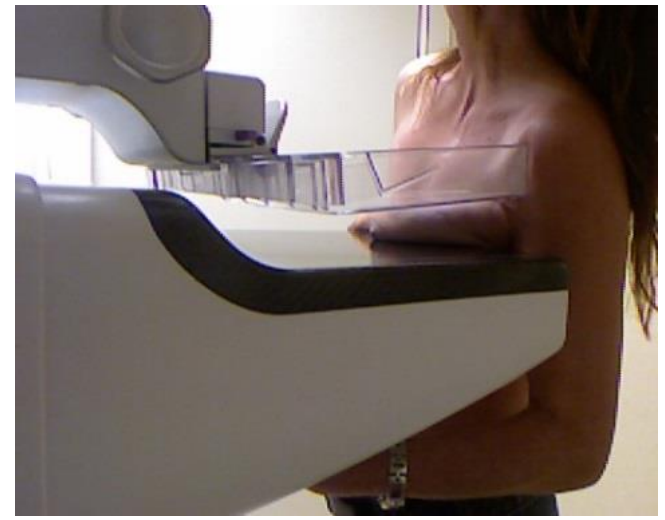

(b)

Fig. 2. (a) Clinical set up including the Kinect sensor (red circle). (b) shows a sample RGB image of a volunteer (compressed breast) acquired by the Kinect sensor.

\section{Results}

The experiment to estimate the Kinect accuracy illustrates high correlation between experimentally and Kinect depth measures (slope $=1.0118, \mathrm{R}^{2}=0.9999$ ), as illustrated in figure 3. Regarding its precision, an average distance error of $3.6 \pm 1.7 \mathrm{~mm}$ was observed. Within the ROI used to measure the Kinect distance, an average variance of 2.7 $\mathrm{mm}$ was found. Therefore, the Kinect sensor produces acceptable results for studying the curvature of the breast.

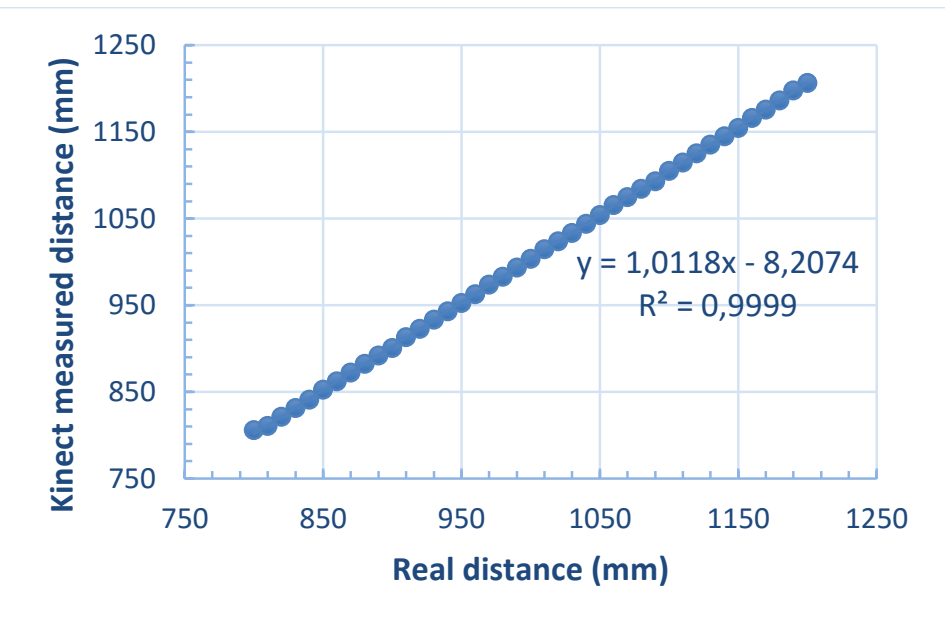

Fig. 3. Distance measured experimentally and using Kinect sensor. 
Sample results using the clinical setting are illustrated in figure 4 for two participants. Three-dimensional triangle meshes of the breast are shown in Figure $4 \mathrm{a}$ and $4 \mathrm{~b}$ for an uncompressed and compressed breast, respectively. Note the tilt of the top surface of the compressed breast due to the flexible compression paddle used during compression. Depth images of the entire scenario and breast are illustrated in Figure $4 c$ and $4 d$, respectively.

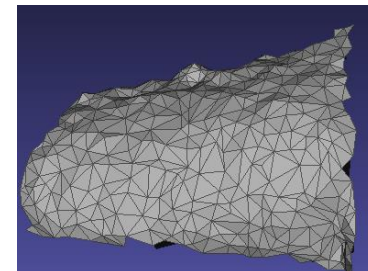

(a) uncompressed breast

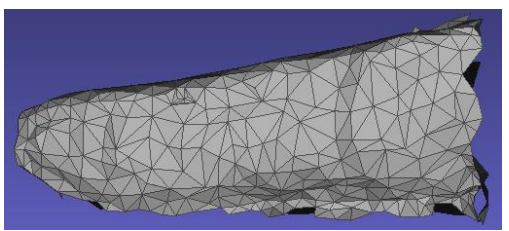

(b) compressed breast

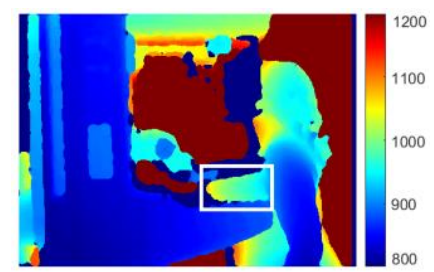

(c) Full depth image compressed

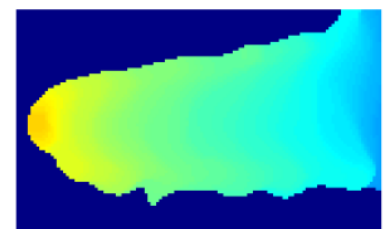

(d) compressed breast

Fig. 4. (a) and (b) correspond to the 3D meshes of an uncompressed and compressed breast, respectively. A full depth image is shown in (c) during compression for a different volunteer. Its corresponding compressed breast (white square) is depicted in (d).

Three-dimensional rendering surfaces of the clinical scenario are shown in figure 5, where both uncompressed and compressed breasts are highlighted in yellow. It can be observed that part of the mammography system, e.g. breast support, is also present.

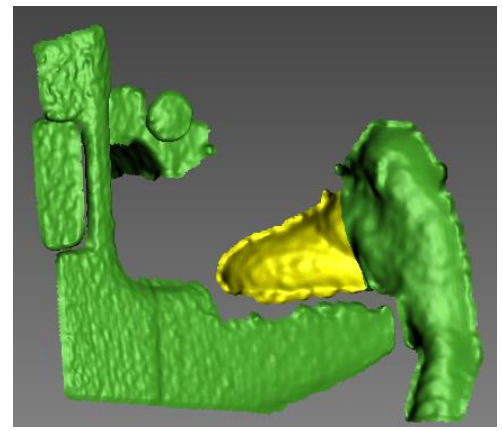

(a) uncompressed position

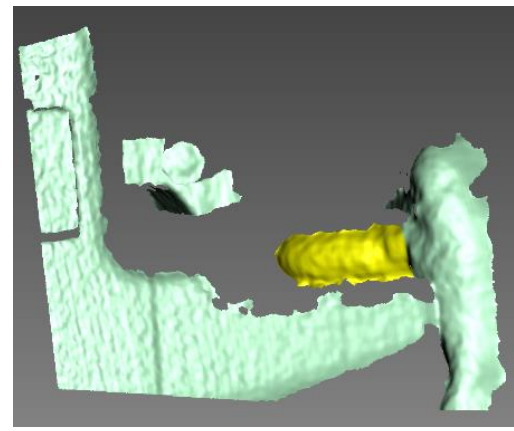

(b) compressed position

Fig. 5. Three-dimensional surface rendering of the clinical scenario for (a) uncompressed and (b) compressed breast. Breasts are highlighted in yellow. Note that part of the mammography system is also displayed. 


\section{Discussion}

Results presented in the previous section illustrate breast surface models with enough spatial information to analyse the deformation of the breast. Initial qualitative analysis demonstrates that the breast shape when compressing with a flexible paddle (figure $4 \mathrm{~b}$ and $4 \mathrm{~d}$ ) is not considered in current anthropomorphic breast models, as observed in figure 1. However due to the small population used in this study, no general conclusions could be made regarding breast shape.

The Kinect sensor used has some limited resolution in the depth map ( 320 x 240 pixels) to capture small details. Therefore, further processing is needed for smoothing the breast models as well as user interaction to correct of imperfections. This has motivated a small experiment with the latest version of the Microsoft Kinect for Windows, i.e. Kinect v2. Figure 6 illustrates the breast surface captured by the Kinect v2 of an uncompressed and compressed breast.

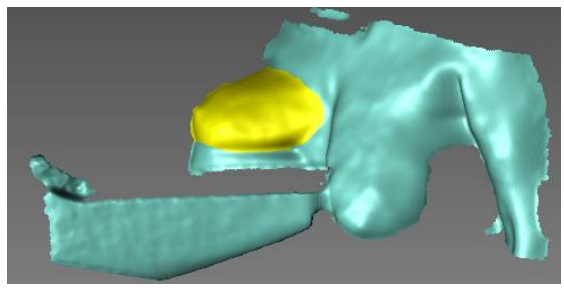

(a) uncompressed position

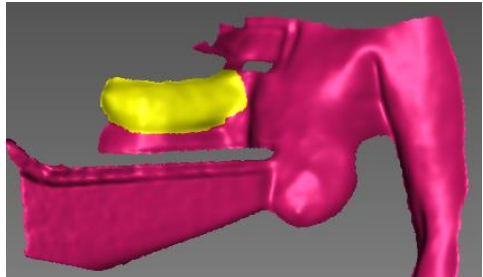

(b) compressed position

Fig. 6. Three-dimensional surface rendering capture by the Kinect sensor v2. (a) Uncompressed and (b) compressed breasts are highlighted in yellow. Note that part of the mammography system is also shown.

Compared with data from Kinect v1 (figure 5), the Kinect v2 sensor shows higher details of the breasts due to the improved depth image resolution (512 x 424 pixels). In addition to this, Kinect v2 sensor has wider horizontal and vertical field of view, which enable to capture more information in a single shot. However, the reflexion of the breast in the breast support adds inaccuracies to the reconstructed breast surface model. Also the transparency of the compression paddle might produce distortions in the top surface of the breast, creating a large hole.

One of the main limitations of this study was the analysis of only craniocaudal compression. The fixed position of the Kinect sensor in a tripod made difficult the capture of the breast for additional views, since the entire system rotates 45 degrees for mediolateral oblique (MLO) and 90 degrees for mediolateral (ML) view. A camera device mounted in the system as used by Tyson et al [14] for breast thickness estimation might be a solution to capture breast surface images in more than one view. 
Another limitation was the use of a single camera sensor. This setup might be sufficient to capture the breast contour, but two calibrated cameras must be employed to capture the entire breast organ as described by Agasthya and Sechopoulos [9].

\section{Conclusions}

This paper investigated an imaging framework which employs a Microsoft Kinect sensor to study breast shape during mammography compression. This has potential to be used for further analysis on breast deformation and eventually produce more accurately compression algorithms. This might lead to more realistic synthetic images in VCTs.

Although several limitations were previously described, the Kinect sensors provide depth images with enough resolution to investigate breast surface. Another potential application of such framework includes quality assessment of patient position to avoid repeated tests during mammography acquisition.

Further work will include the design of a more robust imaging framework which will allow to capture breast surface from a larger number of women, and analyse the possible correlation of breast compression with breast density and size. This will improve previously developed breast compression models developed within our research group [15]. Also, further modifications are needed to account for additional view compressions, i.e. MLO and ML views.

Acknowledgments. This work is part of the SCARtool project (H2020-MSCA-IF2014, reference 657875), a research funded by the European Union within the Marie Sklodowska-Curie Innovative Training Networks. Also, some of the authors have been partially supported from the Ministry of Economy and Competitiveness of Spain, under project references TIN2012-37171-C02-01 and DPI2015-68442-R, and the FPI grant BES-2013-065314. The authors would also like to thank radiographers at UDIAT for their help during image acquisition.

\section{References}

1. Lång, K., Andersson, I., Rosso, A., Tinberg, A., Timberg, P., Zackrisson, S.: Performance of one-view breast tomosynthesis as a stand-alone breast cancer screening modality: results from the Malmö Breast Tomosynthesis Screening Trial, a population-based study. European Radiology 26(1), 184-190 (2015)

2. Ciatto, S., Houssami, N., Bernardi, D. et al.: Integration of 3D digital mammography with tomosynthesis for population breast-cancer screening (STORM): a prospective comparison study. Lancet Oncol 14(7), 583-589 (2013).

3. Skaane, P., Bandos, A.I., Gullien, R. et al.: Comparison of digital mammography alone and digital mammography plus tomosynthesis in a population-based screening program. Radiology 267(1), 47-56 (2013). 
4. Tingberg, A., Fornvik, D., Mattsson, S., Svahn, T., Timberg, P., Zackrisson, S.: Breast cancer screening with tomosynthesis - initial experiences. Radiat. Prot. Dosimetry 147(1-2), 180-183 (2011).

5. Hsu, C.M.L., Palmeri, M.L., Segars, W.P., Veress, A.I., Dobbins, J.T.: An analysis of the mechanical parameters used for finite element compression of a high-resolution 3D breast phantom. Medical Physics 38 (10), 5756-5770 (2011)

6. Bliznakova, K., Suryanarayanan, S., Karellas, A., Pallikarakis, N.: Evaluation of an improved algorithm for producing realistic 3D breast software phantoms: Applications for mammography. Medical Physics 37(11), 5604-5617 (2010)

7. Bakic, P.R., Zhang, C., Maidment, A.D.A.: Development and characterization of an anthropomorphic breast software phantom based upon region-growing algorithm. Medical Physics 38(6), 3165-3176 (2011)

8. Li, C.M., Segars, W.P., Tourassi, G.D., Boone, J.M., Dobbins, J.T.: Methodology for generating a 3D computerized breast phantom from empirical data, Medical Physics 36(7), 3122-3131 (2009)

9. Agasthya, G., Sechopoulos I.: Analysis of the 3-D Shape of Patients' Breast for Breast Imaging and Surgery Planning. AAPM (2015)

10. Pöhlmann, S.T.L., Hewes, J.,Williamson, A. I., Sergeant, J.C., Hufton, A., Gandhi, A., Taylor, C. J., Astley, S.M.: Breast volume measurement using a games console input device. In: Hiroshi, F., Takeshi, H., Muramatsu, C. (eds.) Breast Imaging: 12th International Workshop, IWDM 2014. LNCS, vol. 8539, pp. 666-673. Springer, Heidelberg (2014)

11. Wheat, J.S., Choppin, S., Goyal, A.: Development and assessment of a Microsoft Kinect based system for imaging the breast in three dimensions. Medical Engineering and Physics 36, $732-738$ (2014)

12. Henseler, H., Kuznetsova, A., Vogy, P., Rosenhahn, B.: Validation of the Kinect device as a new portable imaging system for three-dimension breast assessment. Journal of Plastic, Reconstructive and Aesthetic Surgery 67, 483-488 (2014)

13. Díaz, O., Dance, D.R., Young, K.C., Elangovan, P., Bakic, P.R., Wells, K.: Estimation of scattered radiation in digital breast tomosynthesis. Physics in Medicine and Biology 59 (15), 4375-4390 (2014)

14. Tyson, A.H., Mawdsley, G.E., Yaffe, M.J.: Measurement of compressed breast thickness by optical stereoscopic photogrammetry. Medical Physics 36 (2), 569-576 (2009)

15. García, E., Oliver, A., Díez, Y., Díaz, O., Georgii, J., Martí, R., Martí, J.: Comparing regional breast density using Full-Field Mammograms and Magnetic Resonance Imaging: A preleminary study. In: MICCAI-BIA 2015, Proceeding of the 3rd MICCAI Workshop on Breast Image Analysis, 33-40 (2015) 